\title{
Euler-Heisenberg waves propagating in a magnetic background
}

\author{
Elda Guzman-Herrera ${ }^{\mathrm{a}}$, Nora Breton ${ }^{\mathrm{b}}$ \\ Departamento de Física, Centro de Investigación y de Estudios Avanzados del I. P. N., Apdo. 14-740 Mexico City, CDMX, Mexico
}

Received: 20 August 2020 / Accepted: 20 December 2020 / Published online: 3 February 2021

(C) The Author(s) 2021

\begin{abstract}
We derive the Euler-Heisenberg solutions that describe electromagnetic waves propagating through very intense uniform magnetic or electric background, with the effective metric approach. We first explore the case of a magnetic background: as a result of the interaction between the wave and the background there is birefringence and a longitudinal electric field component arises. The two phase velocities depend on the intensity of the external magnetic field and on the polarization of the wave; phase velocities can be slowed down up to the order of hundred thousandths for fields $B / B_{\text {cr }}<<1$. The analogous study is done when the wave propagates through a uniform electric field. We then consider the situation when the background is in movement by means of a Lorentz boost, modeling then a magnetized flowing medium. We determined how this motion affects the speed of propagation of the electromagnetic wave, in this case the phase velocities depend on both the magnetic background and the direction and velocity of the boost.
\end{abstract}

\section{Introduction}

In the presence of intense electromagnetic fields quantum electrodynamics $(\mathrm{QED})$ predicts that vacuum has properties of a material medium as a consequence of the electromagnetic field self-interactions. When the electromagnetic field strengths approach the critical fields $E_{c r} \approx m_{e}^{2} c^{3} /(e \hbar) \approx$ $10^{18} \mathrm{~V} / \mathrm{m}$ or $B_{c r} \approx 10^{9} \mathrm{~T}$ the impact of the external field on quantum processes becomes significant.

The Euler-Heisenberg (EH) Lagrangian was derived from QED principles by Heisenberg and Euler [1]. For a nice discussion on the history of the Euler-Heisenberg approach see [2] and a pedagogical review can be found in [3]. By treating the vacuum as a medium, EH effective action predicts nonlinear light interaction processes since it takes into account

\footnotetext{
a e-mail: eguzman@ fis.cinvestav.mx

b e-mail: nora@fis.cinvestav.mx (corresponding author)
}

vacuum polarization to one loop, and is valid for electromagnetic fields that change slowly compared to the inverse electron mass. The EH Lagrangian $\mathcal{L}_{\mathrm{EH}}(F, G)$ depends in nonlinear way on the two Lorentz and gauge invariants of the Faraday tensor $F_{\mu \lambda}, F=F^{\mu \lambda} F_{\mu \lambda}=2\left(B^{2}-E^{2}\right)$ and $G=F^{* \mu \lambda} F_{\mu \lambda}=-4 \vec{B} \cdot \vec{E}$, with the dual field $F^{* \mu \lambda}=$ $\frac{1}{2} \epsilon^{\mu \lambda \alpha \beta} F_{\alpha \beta}$,

$$
\begin{aligned}
& \mathcal{L}_{\mathrm{EH}}(F, G)=-\frac{F}{4}-\frac{1}{8 \pi^{2}} \int_{0}^{\infty} \\
& \times e^{-m^{2} s}\left[(e s)^{2} \frac{\operatorname{Re}[\cosh (e s \sqrt{2(F+i G)})]}{\operatorname{Im}[\cosh (e s \sqrt{2(F+i G)})]}\right. \\
& \left.\quad-\frac{2}{3}(e s)^{2} F-1\right] \frac{d s}{s^{3}} .
\end{aligned}
$$

From this Lagrangian new nonlinear interactions can be derived, which do not occur in the tree level Maxwell action; among them are light-light interaction and pair production from vacuum excited by an electromagnetic field. It has been thoroughly investigated, and higher loop contributions in strong fields have been calculated as well, see for instance $[4,5]$.

The Lagrangian (1) can be expanded into an asymptotic series [6,7] whose first terms, of the order $\alpha^{2}$, are

$\mathcal{L}_{\mathrm{EK}}(F, G)=-\frac{F}{4}+\frac{\mu}{4}\left(F^{2}+\frac{7}{4} G^{2}\right)$,

where $\mu$ is the parameter of the EH theory that in terms of the fine structure constant, $\alpha(c=1, h=1)$, is

$\mu=\frac{2 \alpha^{2}}{45 m_{e}^{4}}$

that in terms of the critical fields, is of the order $\mu \sim \alpha / B_{c r}^{2}$. Actually (2) is the Euler-Kockel (EK) Lagrangian. Euler and Kockel, two Heisenberg's students, investigated on QED vacuum polarizations in the constant background limit, obtaining the leading nonlinear correction in powers of the field 
strengths, presenting the Lagrangian (2) in 1935 [8]. To describe the propagation of photons in an external field in QED only this first correction is needed, i.e. the term $2 \alpha^{2}\left(F^{2}+7 G^{2} / 4\right) /\left(45 m_{e}^{4}\right)$. The use of the first terms of this expansion is justified if the dimensionless expansion parameter $4 \pi \alpha \hbar^{3}|F|^{2} /\left(m_{e}^{4} c^{5}\right)$ is much smaller than unity [9]. This is indeed the case even for strong magnetic fields, for instance the magnetic fields in neutron stars that may be as large as $10^{12}$ Gauss [10], then processes like photon splitting and pair conversion are expected to occur in the vicinity of neutron stars [11].

We also note related work by Born and Infeld [12], who obtained similar nonlinear corrections to Maxwell theory but from a classical perspective. See [13] for a generalized BornInfeld electrodynamics. The linear electromagnetic Maxwell theory is recovered if $\mu=0, \mathcal{L}_{\text {Maxwell }}(F)=-F / 4$.

Vacuum polarization effects [14-16] have been studied since the establishment of these Lagrangians and experimental efforts are currently in progress for measuring some of these nonlinear effects, we mention just a few of them: Light by light interactions can be studied using heavy-ion collisions; the electromagnetic (EM) field strengths produced, for example by a $\mathrm{Pb}$ nucleus would be up to $10^{25} \mathrm{~V} \mathrm{~m}^{-1}$, those intense EM fields can be treated as a beam of quasi-real photons, and it has been measured light by light scattering in $P b+P b$ collisions at the Large Hadron Collider [17]. Other experimental evidences include the measurement of photon splitting in strong magnetic fields [18] and the search for vacuum polarization with laser beams crossing magnetic fields or the detection of vacuum birefringence with intense laser pulses [19]. There is also the detection of QED vacuum nonlinearities using waveguides [20]. Vacuum pair production, known as the Sauter-Schwinger effect [14], was a prediction in the $1936 \mathrm{EH}$ paper however the necessary electric field strengths are not reached yet, since the corresponding critical laser intensity is about $I_{\mathrm{cr}}=4.3 \times 10^{29} \mathrm{~W} / \mathrm{cm}^{2}$ [21].

The phase velocity of an electromagnetic wave traveling through intense EM fields will be altered due to vacuum polarization. It is also well known the emergence of a longitudinal field component, as well as the decrease of the phase velocity $[6,9,22]$. Another effect that arises in strong magnetic backgrounds is the birefringence [23]. In order to attempt the detection of nonlinear electromagnetic effects it is crucial to determine the velocity of propagation of the electromagnetic wave in the intense EM background, and the aim of our paper is to determine the phase velocities that correspond to the birefringence as well as the electric field longitudinal component in terms of the magnetic background derived from the Euler-Kockel Lagrangian. Although these effects have been the subject of many studies, our approach is the framework of the effective metrics derived in nonlinear electrodynamics for curved spaces.

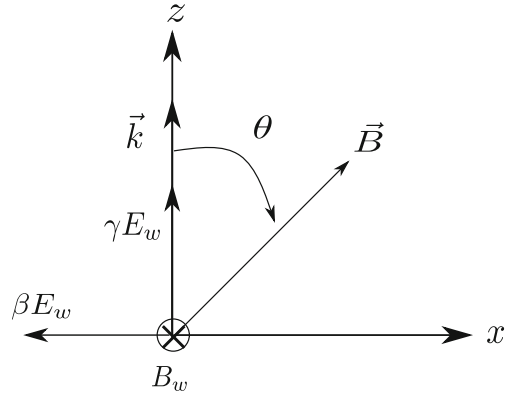

Fig. 1 Plane wave electromagnetic fields with the electric component $\gamma E_{w}(\xi)$ arising in the $\hat{z}$ direction, due to the nonlinear interaction. The uniform magnetic field background is shown making a $\theta$ angle with the $z$-axis.The magnitude of the fields is not on scale

The paper is organized as follows: In the next section the equations derived from the Euler-Kockel Lagrangian (2) are presented and the electromagnetic fields of the background and the wave are introduced; then by means of the effective metric approach we determine the birefringence and the two effective metrics, whose null geodesics are the light trajectories, determining then the corresponding phase velocities, the polarizations and the dispersion relations; in the same section we present the results when the background is a uniform electric field. In Sect. 3, by performing a Lorentz boost, we consider the background in movement, determining the velocity of the modeling medium and the changes that this situation introduces in the phase velocities of the propagation. Finally, conclusions are presented in the last section.

\section{The Euler-Heisenberg propagating wave}

In this section we derive the solutions to the EK field equations. The nonlinear electrodynamics (NLED) field equations derived from the EK Lagrangian (2), are

$F_{\lambda \mu ; \nu}+F_{\nu \lambda ; \mu}+F_{\mu \nu ; \lambda}=0$

$\partial_{\nu}\left[\sqrt{-g}\left(\mathcal{L}_{\mathrm{F}} F^{\mu \nu}+\mathcal{L}_{\mathrm{G}} F^{* \mu \nu}\right)\right]=0$,

where $\mathcal{L}_{\mathrm{X}}$ denotes the derivative of $\mathcal{L}$ with respect to the invariant $X, d \mathcal{L} / d X$.

Due to the interaction between the wave and the background, it is known that the parallel mode of the propagating wave fails to be orthogonal to the wave vector $\vec{k}$ [23], because a longitudinal wave component arises in the presence of strong magnetic fields.

We shall consider an electromagnetic (EM) wave propagating through a uniform magnetic background. The wave fields are function of $\xi=(z-v t)$, where $v$ is the phase velocity of the propagation in the $\hat{z}$ direction; the electric and magnetic fields of the propagating wave in terms of the 
electromagnetic potential $a^{\mu}$ are proposed as

$a_{\mu}(\xi)=(0, a(\xi), 0,-\gamma a(\xi) / v)$.

We consider the arising electric component being $\gamma a(\xi)$, and the constant $\gamma$ is to be determined by solving the nonlinear electrodynamics (NLED) field Eq. (4). The propagating vector $\vec{k}$ is along the $\mathrm{z}$-direction, $k^{\mu}=(\omega, 0,0, k)$, the wave electric field is along the $x$-direction and the wave magnetic field points to the $y$-direction. While for the uniform background $A^{\mu}$

$$
A_{\mu}(\xi)=\left(0,0, B_{x} z-B_{z} x, B_{y} x\right)
$$

In Fig. 1 is shown the plane wave EM fields propagating in the magnetic background; for convenience we locate the magnetic background in the plane XZ, $\vec{B}=B \sin \theta \hat{x}+$ $B \cos \theta \hat{z}=B_{x} \hat{x}+B_{z} \hat{z}$.

The photon polarization tensor in a homogeneous electromagnetic background in the context of QED has been addressed in [24], and in [25] was studied the case of a BornInfeld wave in a magnetic background. In the next subsection we determine the phase velocities $v_{i}, i=1,2$ and $\gamma$ in the effective metric approach.

\subsection{Effective metric and phase velocities of light rays}

It is well known that the intense EM fields can resemble a curved spacetime, in the sense that light trajectories are not straight lines but suffer deflection. Deviations from the straight trajectories in vacuum are described in NLED by the null trajectories of an effective metric. According to this approach [26-28], the magnetic background distorts the spacetime where the EM wave propagates and considering the propagation as a perturbation, (i. e. the EM fields of the propagating wave are much smaller than the background fields) the effective metric is derived from the analysis of the propagation of the discontinuities of the field or characteristic surfaces (wavefronts). If $k_{\mu}$ is a null vector normal to the characteristic surface of the wave, the effective metric $g_{\text {eff }}^{\mu \nu}$ is given by

$g_{\text {eff }}^{(i) \mu v} k_{\mu} k_{v}=0, \quad i=1,2$.

The (i) superscript corresponds to the two metrics that can arise in NLED, where the phenomenon of birefringence can occur. See [29] for a study on the Fresnel equation in nonlinear electrodynamics and [30] for a classification of the effective metrics.

Equations (7) are actually the dispersion relations, that for a wave number $k^{\mu}=(\omega, 0,0, k)$ amount to

$$
g_{\mathrm{eff}}^{(i) t t} \omega^{2}-2 g_{\mathrm{eff}}^{(i) t z} \omega k+g_{\mathrm{eff}}^{(i) z z} k^{2}=0
$$

$$
\begin{aligned}
v_{i} & =\left(\frac{\omega}{k}\right)_{i}=-\frac{g_{\text {eff }}^{(i) t z}}{g_{\text {eff }}^{(i) t t}} \pm \sqrt{\left(\frac{g_{\text {eff }}^{(i) t z}}{g_{\text {eff }}^{(i) t t}}\right)^{2}-\left(\frac{g_{\text {eff }}^{(i) z z}}{g_{\text {eff }}^{(i) t t}}\right)}, \\
i & =1,2
\end{aligned}
$$

where $v_{i}$ is the phase velocity of the propagation. The equations of the propagation of the field discontinuities in nonlinear electrodynamics characterized by a Lagrangian $\mathcal{L}(F, G)$ are given in [28] [Eqs. (16) in [28]]; in case of the EK Lagrangian, $\mathcal{L}_{F G}=0$ and the effective metrics (7) are given by,

$g_{\text {eff }}^{(1) \mu \nu}=\left(\mathcal{L}_{F}-2 \mathcal{L}_{G G} F\right) \eta^{\mu \nu}-4 \mathcal{L}_{G G} F_{\lambda}^{\mu} F^{\lambda \nu}$,

$g_{\text {eff }}^{(2) \mu \nu}=\mathcal{L}_{F} \eta^{\mu \nu}-4 \mathcal{L}_{F F} F^{\mu}{ }_{\lambda} F^{\lambda v}$,

where $\eta^{\mu \nu}=\operatorname{diag}[+1,-1,-1,-1]$ is the Minkowski metric. In the Maxwell case $\mathcal{L}=-F / 4, \mathcal{L}_{F}=-1 / 4, \quad \mathcal{L}_{F F}=$ 0 and $\mathcal{L}_{G}=0$, then both effective metrics become conformal to the Minkowski metric, $g_{\text {eff }}^{(1)}=g_{\text {eff }}^{(2) \mu \nu}=-\eta^{\mu \nu} / 4$, and the null geodesics coincide with the Minkowski ones. The nonvanishing electromagnetic tensor components of the magnetic background are $F^{x y}=-B_{z}, F^{x z}=B_{y}=0$, $F^{y z}=-B_{x}$, and $F=2 B^{2}$, the corresponding phase velocities $v_{i}$ derived from the two effective metrics (10) and (11), through Eq. (9) are

$\left(v_{1}\right)^{2}=1-\frac{14 \mu B_{x}^{2}}{1+10 \mu B^{2}} \approx 1-14 \mu B_{x}^{2}$,
$\left(v_{2}\right)^{2}=1-\frac{8 \mu B_{x}^{2}}{1-4 \mu B^{2}} \approx 1-8 \mu B_{x}^{2}$,

where the last line corresponds to the expressions up to quadratic order in the background field.

The effective metric approach turns out to be equivalent to a soft photon approximation. Splitting the total electromagnetic field into the background field $\vec{B}$ and the propagating photon $f_{\mu \nu}$,

$f_{\mu \nu}=\partial_{\mu} a_{\nu}-\partial_{\nu} a_{\mu}=\left(\epsilon_{\mu} k_{\nu}-\epsilon_{\nu} k_{\mu}\right) e^{-i k x}$,

with the vector potential $a^{\mu}(k)$, the polarization $\epsilon_{\mu}=$ $a_{\mu} / \sqrt{a^{\alpha} a_{\alpha}}$, the wave vector $k^{\mu}=(\omega, 0,0, k)$, and being the phase $k x=k^{\alpha} x_{\alpha}=-k \xi$. Keeping the linear approximation with respect to $f_{\mu \nu}$ in the equations of motion, leads to an eigenvalue equation for the propagating modes $[31,32]$

$A^{\mu v} \epsilon_{\nu}=0$

where $A^{\mu v}$ is given by

$$
\begin{aligned}
A^{\mu \nu}= & c_{1} F^{\mu \alpha} F^{\nu \beta} k_{\alpha} k_{\beta}+c_{2} F^{* \mu \alpha} F^{* \nu \beta} k_{\alpha} k_{\beta} \\
& +c_{3}\left(\delta^{\mu \nu} \kappa^{2}-k^{\mu} k^{\nu}\right), \\
c_{1}= & \frac{1}{2} \mathcal{L}_{F F}, \quad c_{2}=\frac{1}{2} \mathcal{L}_{G G}, \quad c_{3}=\frac{1}{2} \mathcal{L}_{F},
\end{aligned}
$$


where $\kappa=k^{\alpha} k_{\alpha}=\omega^{2}-k^{2}, k^{\mu}=(\omega, 0,0, k)$. Eq. (15) is the light cone condition and its solutions are the dynamically allowed polarization modes. Moreover, adopting the temporal gauge $\epsilon_{0}=0$, then Eq. (15) splits into

$A^{0 i} \epsilon_{0}=0, \quad A^{i j} \epsilon_{j}=0$.

For the case under study, the nonvanishing electromagnetic tensor components of the magnetic background are $F^{x y}=-B_{z}, F^{x z}=B_{y}=0, F^{y z}=-B_{x}$, and their dual $F^{* t x}=-B_{x}, F^{* t y}=-B_{y}=0, F^{* t z}=-B_{z}$ and $F=2 B^{2}$. Explicitly Eqs. (18) become

$$
\begin{aligned}
& c_{2} B_{z} B_{x} \epsilon_{1}+\left(c_{2} B_{z}^{2}-c_{3}\right) \epsilon_{3}=0, \\
& {\left[c_{2} \omega^{2} B_{x}^{2}-c_{3}\left(\omega^{2}-k^{2}\right)\right] \epsilon_{1}+c_{2} \omega^{2} B_{z} B_{x} \epsilon_{3}=0,} \\
& {\left[c_{1} k^{2} B_{x}^{2}-c_{3}\left(\omega^{2}-k^{2}\right)\right] \epsilon_{2}=0,}
\end{aligned}
$$

The Eq. $A^{0 i} \epsilon_{0}=0$ turns out to be the same than Eq. (19). Note that in Eqs. (19) and (20) are coupled $\epsilon_{1}$ and $\epsilon_{3}$; these equations define the parallel polarization tensor as $\epsilon_{\|}=$ $[0, a(\xi) / k a, 0,-\gamma a(\xi) /(\omega a)]$ or $\epsilon_{\|}=[0,1,0,-\gamma / v]$ and determine the arising electric component $\gamma$ and a first dispersion relation as

$$
\begin{aligned}
& \gamma=\frac{14 \mu B_{x} B_{z}}{1-4 \mu B^{2}+14 \mu B_{z}^{2}} v_{1} \approx 14 \mu B_{x} B_{z}+O\left(B^{4}\right), \\
& \left(\frac{\omega}{k}\right)_{(1)}^{2}=\left(v_{1}\right)^{2}=1-\frac{14 \mu B_{x}^{2}}{1+10 \mu B^{2}} \\
& \quad \approx 1-14 \mu B_{x}^{2}+O\left(B^{4}\right) .
\end{aligned}
$$

The angle $\delta$ between the polarization $\epsilon_{\|}$and the the propagating vector $\vec{k}$, is

$$
\delta=\operatorname{arccot}\left(\frac{\gamma}{v}\right)=\frac{14 \mu B_{x} B_{z}}{1-4 \mu B^{2}+14 \mu B_{z}^{2}} .
$$

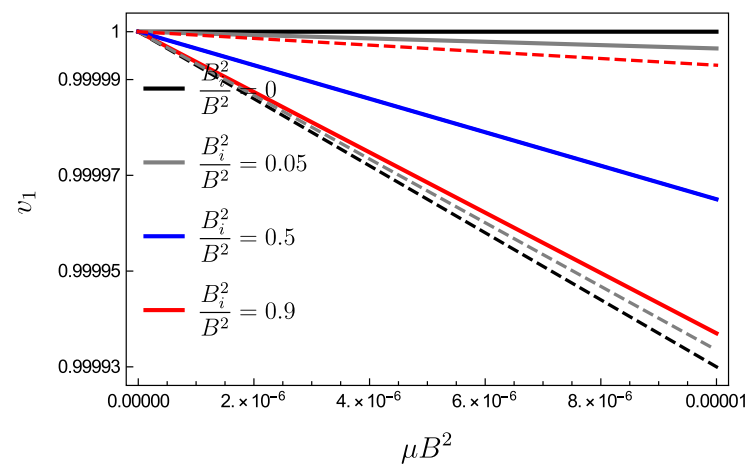

Fig. 2 The phase velocities $v_{1}, v_{2}$ are shown as a function of the (dimensionless) magnetic background $\mu B^{2}$, for four values of $\left(B_{x} / B\right)^{2}$ (thick lines) and $\left(B_{z} / B\right)^{2}$ (dashed lines). As $\mu B^{2}$ increases $v_{1,2}$ diminish. If $\mu B^{2}=0$ the velocity is the one in vacuum, $v_{i}=c=1$. Note that if $\left(B_{x} / B\right)^{2}<0.5$ the slowing effect increases as the transver-
While Eq. (21) does not impose any condition on $\epsilon_{2} \neq 0$, then we can set the transversal polarization mode as $\epsilon_{\perp}=$ $(0,0,1,0)$, and the second dispersion relation is

$$
\left(\frac{\omega}{k}\right)_{(2)}^{2}=\left(v_{2}\right)^{2}=1-\frac{8 \mu B_{x}^{2}}{1-4 \mu B^{2}} \approx 1-8 \mu B_{x}^{2}+O\left(B^{4}\right)
$$

where $B^{2}=B_{x}^{2}+B_{z}^{2}$. Transversal and parallel are defined with respect to the plane spanned by the magnetic field $B$ and the wave number $\vec{k}$, that is the plane $X Z$. Phase velocities are in agreement with the ones derived from the effective metrics given by (10) and (11).

As a consequence of the nonlinear interaction wavebackground there is a retarding term in $v_{i}$ that depends on the background field; specifically the retarding term arises due to the magnetic component that is perpendicular to the propagating direction, $B_{x}=B_{\perp}$, in such a way that if $B_{\perp}=0$, then the velocity is the one in vacuum.

The phase velocities $v_{\mathrm{i}}, i=1,2$ of the propagation through the magnetic background, Eqs. (23), (25), are illustrated as a function of the dimensionless $\mu B^{2}$ in Fig. 2. The truncation we have used to describe the propagation of photons in an external field is indeed valid for strong magnetic fields that may be as large as $10^{12}$ Gauss $\left(10^{-1}\right.$ Tesla), that compared to the critic field $B_{\mathrm{cr}} \sim 10^{9}$ Tesla, gives us the range of validity of our variable $\mu B^{2} \sim \alpha\left(B / B_{\mathrm{cr}}\right)^{2}$, and considering that $B / B_{\mathrm{cr}} \sim 1 / 100$, the range of validity is $\mu B^{2} \in\left[0,10^{-6}\right)$. In agreement to this range the slowing down of the wave is on the hundred of thousandths. As shown in Fig. 2 it would be very difficult to distinguish in an experiment if birefringence occurs. The slowing down depends on the relative magnitude of $B_{x}$ and $B_{z}$; if $\left(B_{x} / B\right)^{2}<0.5$ the slowing effect increases as the transversal magnetic component $B_{x}$ does; while if $\left(B_{z} / B\right)^{2}<0.5$ the slowing effect

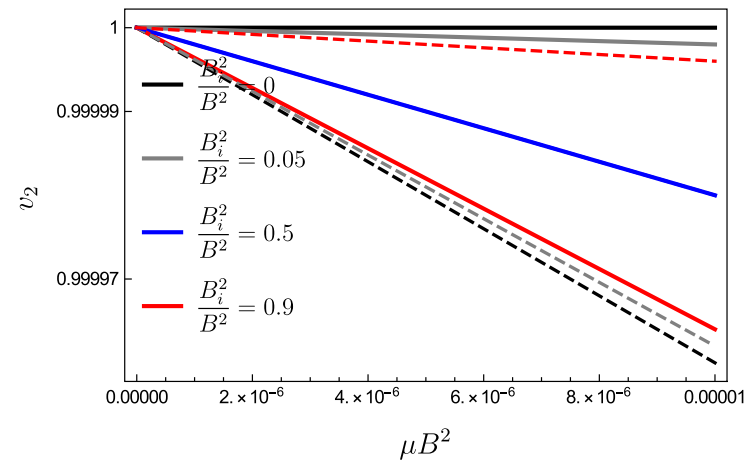

sal magnetic component $B_{x}$ does; while if $\left(B_{z} / B\right)^{2}<0.5$ the slowing effect increases as the parallel magnetic component $B_{z}$ does. If $\left(\frac{B_{x}}{B}\right)^{2}=\left(\frac{B_{z}}{B}\right)^{2}=\frac{1}{2}$, the corresponding velocities $v_{1}$ and $v_{2}$ are equal (blue line) 


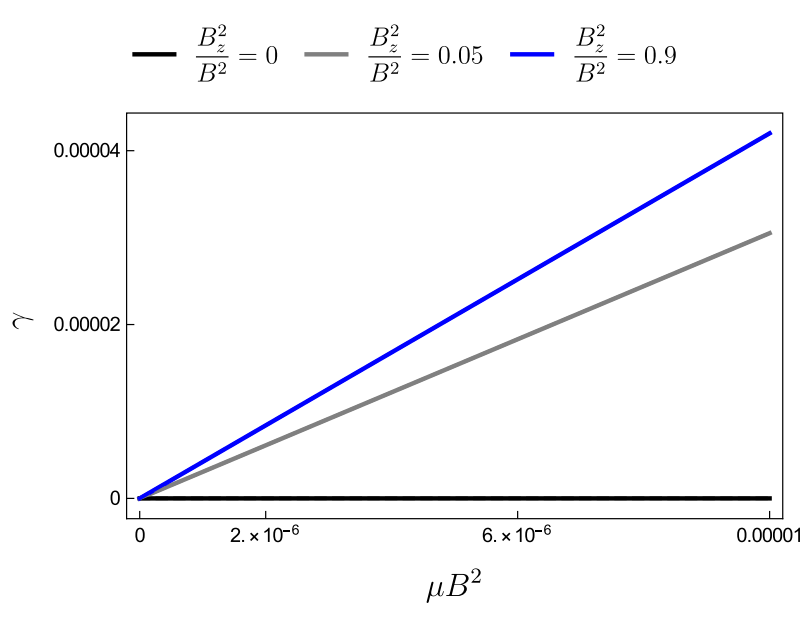

Fig. 3 It is plotted $\gamma$, the arising wave electric component in the propagating direction, as a function of $\mu B^{2}$ for different values of $B_{z}^{2} / B^{2}$

increases as the parallel magnetic component $B_{z}$ does. If $\left(\frac{B_{x}}{B}\right)^{2}=\left(\frac{B_{z}}{B}\right)^{2}=\frac{1}{2}$, the corresponding velocities $v_{1}$ and $v_{2}$ become the same. In Fig. 3 it is illustrated the factor $\gamma$ of the arising wave electric component in the propagating direction.

In order that $v_{2}$ Eq. (25) be a real number, a lower bound arises for the magnetic field $B, \mu B^{2} \leq 1 / 4$, that in terms of the critical magnetic field $B_{c r}$ is $\mu B^{2} \approx \alpha\left(B / B_{c r}\right)^{2}$, since $\left(B / B_{c r}\right)<<1$ that value is never reached in this approximation. If $\mu=0$, that means the absence of vacuum polarization then the light velocity in vacuum, $v=c$, is recovered.

\subsection{The electric uniform background}

In an analogous way to the previous subsection we now consider an electric background. The propagation of an EM wave through an intense uniform electric field is of interest [6] since there is the prediction of vacuum electron-positron production that has not yet been measured, however it might be feasible in the near future, due to the high power reached lately by lasers $[33,34]$.

In this case the nonvanishing electromagnetic tensor components for the electric background are $F^{t x}=-E_{x}, F^{t y}=$ $-E_{y}, F^{t z}=-E_{z}$, and their dual $F^{* x y}=E_{z}, F^{* x z}=$ $-E_{y}=0, F^{* y z}=E_{x}$, and $F=-2 E^{2}=-2\left(E_{x}^{2}+E_{z}^{2}\right)$. The resulting equations from (18) have the form of Eqs. (19)(21) interchanging $c_{1} \leftrightarrow c_{2}$ and $B_{i} \leftrightarrow E_{i}$. Solving them we get the following $\gamma$-component and the phase velocities

$$
\begin{aligned}
\gamma & =\frac{8 \mu E_{x} E_{z}}{1+4 \mu E^{2}+8 \mu E_{z}^{2}} v_{1} \approx 8 \mu E_{x} E_{z}, \\
\left(\frac{\omega}{k}\right)_{(1)}^{2} & =\left(v_{1}\right)^{2}=1-\frac{14 \mu E_{x}^{2}}{1+4 \mu E^{2}} \approx 1-14 \mu E_{x}^{2}, \\
\left(\frac{\omega}{k}\right)_{(2)}^{2} & =\left(v_{2}\right)^{2}=1-\frac{8 \mu E_{x}^{2}}{1+12 \mu E^{2}} \approx 1-8 \mu E_{x}^{2} .
\end{aligned}
$$

Phase velocities $v_{1}$ and $v_{2}$ can be derived as well from Eq. (8). $v_{1}$ and $v_{2}$ are shown in Fig. 4; the plots are very similar and for the shown scale there is not much qualitative difference. Analogous observations than for the magnetic background apply to the electric case.

\section{The phase velocity of the EM wave in a flowing magnetic background}

Another interesting situation is when the background is not still but moving with constant velocity; it can be considered as a plasma model, for instance. Any effective metric can be considered as a moving medium, as we show in what follows, as long as the effective metric be nondiagonal. For the case we have studied this means that the metric component $g_{t z}^{\text {eff }}$ be nonvanishing. This we achieve by performing a Lorentz transformation on the NLED effective metric.

\subsection{The effective metric as a Painlevé-Lemaitre-Gullstrand (PLG) metric}

Let us consider a completely general effective metric (nondiagonal); to determine the phase velocity of the EM wave $\beta=v / c$, we calculate the null geodesics of the effective metric, by making zero the line element, $d s^{2}=g_{\mu \nu}^{\text {eff }} d x^{\mu} d x^{\nu}=0$,

$\frac{d s^{2}}{d \tau^{2}}=g_{\mu \nu}^{\text {eff }} \frac{d x^{\mu}}{d \tau} \frac{d x^{\nu}}{d \tau}=g_{\mu \nu}^{\text {eff }} \dot{x}^{\mu} \dot{x^{\nu}}=0$.

Considering Cartesian coordinates $(t, x, y, z)$ and a light trajectory for fixed $x$ and $y,(\dot{x}=0=\dot{y})$, we obtain from Eq. (29) a quadratic equation for the phase velocity along the $z$-direction, $\beta=d z / d t=\dot{z} / \dot{t}$; then solving for $\beta=v / c$ we obtain the phase velocity in terms of the metric components of the effective metric,

$\beta=\frac{d z}{d t}=-\frac{g_{t z}^{\text {eff }}}{g_{z z}^{\text {eff }}} \pm \frac{\sqrt{\left(g_{t z}^{\text {eff }}\right)^{2}-g_{t t}^{\text {eff }} g_{z z}^{\text {eff }}}}{g_{z z}^{\text {eff }}}$.

This expression is in agreement with Eq. (9). The interpretation of the effective metric as a propagating medium can be seen clearly by writing the effective metric $g_{\mu \nu}^{\text {eff }}$ in the form of the Painlevé-Lemaitre-Gullstrand (PLG) metric, that in Cartesian coordinates $(\tilde{t}, \tilde{x}, \tilde{y}, \tilde{z})$ for $\tilde{x}=$ const $\tilde{y}=$ const, is given by

$d s^{2}=-\left(\tilde{c}^{2}-V^{2}\right) d \tilde{t}^{2}-2 V d \tilde{z} d \tilde{t}+d \tilde{z}^{2}$,

where $V(\tilde{t}, \tilde{z})$ represents the velocity of the propagating medium and $\tilde{c}(\tilde{t}, \tilde{z})$ the velocity of the perturbation propagating through such a medium [35]. Taking advantage of the 


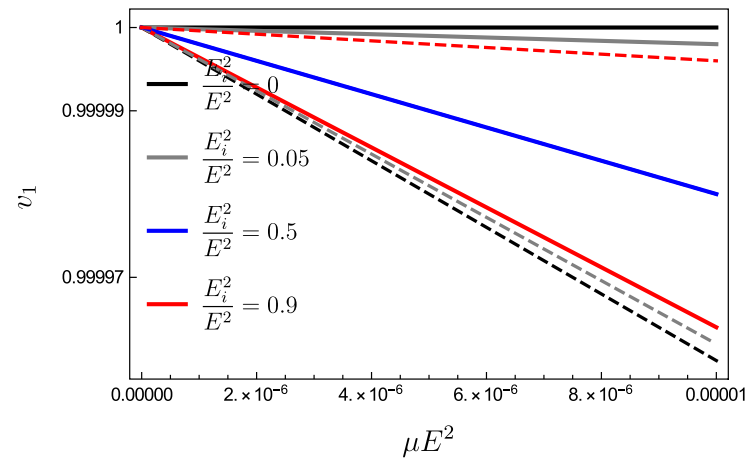

Fig. 4 The phase velocities $v_{i}$ versus $\mu E^{2}$ are displayed. Velocities approach the ones in vacuum as $\mu E^{2} \mapsto 0$. Thick lines correspond to different $\left(E_{x} / E\right)^{2}$ and the dashed lines to $\left(E_{z} / E\right)^{2}$. For the

constant curvature (scalar curvature is zero $R=0$ ) of the effective metric, by making a scale transformation on the $(t, z)$ coordinates we can write the effective metric Eq. (29) in the PLG form (31). By re-scaling as

$t \mapsto \frac{\sqrt{V^{2}-\beta^{2}}}{\sqrt{g_{t t}}} \tilde{t}, \quad z \mapsto \frac{1}{\sqrt{g_{z z}}} \tilde{z}$,

the effective metric in the $(\tilde{t}, \tilde{z})$ coordinates acquires the form,

$d \tilde{s}^{2}=-\left(\beta^{2}-V^{2}\right) d \tilde{t}^{2}+2 g_{t z}^{\text {eff }} \frac{\sqrt{V^{2}-\beta^{2}}}{g_{t t}^{\text {eff }} g_{z z}^{\text {eff }}} d \tilde{z} d \tilde{t}+d \tilde{z}^{2}$,

comparing with (31), we identify the velocity of the perturbation as $\tilde{c}=\beta$ and we determine the velocity of the medium $V$ as

$V= \pm \frac{-g_{t z}^{\text {eff }} \beta}{\sqrt{\left(g_{t z}^{\text {eff }}\right)^{2}-g_{t t}^{\text {eff }} g_{z z}^{\text {eff }}}}$.

Note that if $g_{t z}^{\text {eff }}=0$ then $V=0$, i.e. the medium is static. By performing a Lorentz boost of velocity $\beta_{L}$ in the $z$-direction $\Lambda_{z}$, on the effective metric, $\Lambda_{z} g_{\text {eff }} \Lambda_{z}^{T}=g_{\text {eff }}^{\prime}$, we obtain a nondiagonal metric, that we denote with a prime $g_{\mu \nu}^{\text {eff }}$. The effect of the Lorentz transformation is of mixing the components of the effective metric, in such a way that if the effective metric is diagonal, the transformed one has nondiagonal components, i.e. $g_{t z}^{\text {eff }}$ does not vanish. The original (untransformed) effective metric is recovered when $\beta_{L}=0$. Note as well that the velocity of the medium $V$ is not the same than the one of the Lorentz transformation $\beta_{L}$. The relationship between $V$ and $\beta_{L}$ is given implicitly in Eq. (34), with $g_{\mu \nu}^{\text {eff }} \mapsto g_{\mu \nu}^{\text {eff }}$.

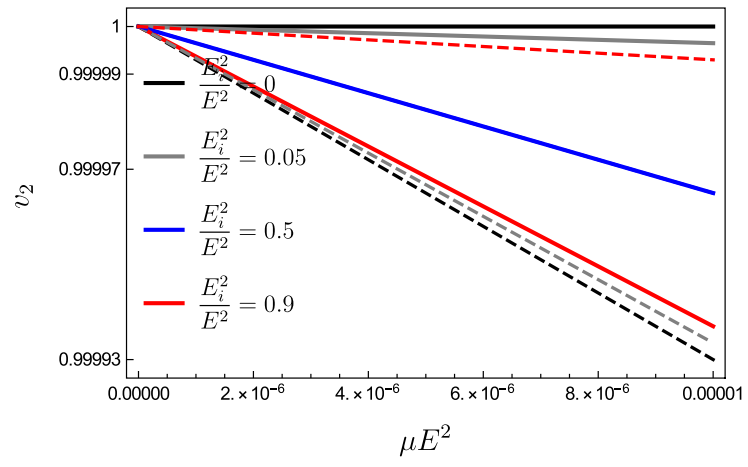

electric uniform background $v_{2}$ reaches lower values than $v_{2}$. When $\left(E_{x} / E\right)^{2}=\left(E_{z} / E\right)^{2}=\frac{1}{2}$ the velocities are the same (thick line overlaps dashed line)

\subsection{The magnetic background Lorentz boosted}

In this subsection we show how the phase velocity is affected when a Lorentz boost is performed on the magnetic background.

Making a Lorentz transformation along the z-direction changes the magnetic background and an electric field component arises: such that now the nonvanishing electromagnetic tensor components of the magnetic background are $F^{t y}=-\gamma_{L} \beta_{L} B_{x}, F^{x y}=-B_{z}, F^{x z}=B_{y}=0, F^{y z}=$ $-\gamma_{L} B_{x}$, and $F=2 B^{2}$.

Calculating the phase velocities using the effective metrics in Eqs. (10) and (11), through Eq. (9) the obtained expressions are cumbersome but being consistent with the approximation up to $O\left(B^{2}\right)$, they simplify to

$$
\begin{aligned}
& v_{1}^{L T z}=1-14 \mu B_{x}^{2}\left(\frac{1+\beta_{L}}{1-\beta_{L}}\right)+O\left(B^{4}\right), \\
& v_{2}^{L T z}=1-8 \mu B_{x}^{2}\left(\frac{1+\beta_{L}}{1-\beta_{L}}\right)+O\left(B^{4}\right),
\end{aligned}
$$

where $\beta_{L}$ is the velocity of the Lorentz transformation along $z$. The retarding term is enhanced due to the factor $\left(1+\beta_{L}\right) /\left(1-\beta_{L}\right)$, that is always greater than one, therefore increasing the slowing down effect on the propagating wave.

Let us now try a Lorentz boost in the direction perpendicular to the propagation of the wave. By transforming the background with a Lorentz boost in the $x$-direction, the magnetic background results as $F^{t y}=\gamma_{L} \beta_{L} B_{z}, F^{x y}=$ $-\gamma_{L} B_{z}, F^{x z}=B_{y}=0, F^{y z}=-B_{x}$; ; in such a way that the phase velocities using the effective metrics in Eqs. (10) and (11), through Eqs. (9), just keeping terms up to $O\left(B^{2}\right)$, are

$v_{1}^{L T x}=1-14 \mu \gamma_{L}^{2}\left[B_{x}^{2}+\beta_{L}^{2}\left(B_{z}^{2}-B_{x}^{2}\right)-2 \beta_{L} B_{x} B_{z} \sqrt{1-\beta_{L}^{2}}\right]$,

$v_{2}^{L T x}=1-8 \mu \gamma_{L}^{2}\left[B_{x}^{2}+\beta_{L}^{2}\left(B_{z}^{2}-B_{x}^{2}\right)-2 \beta_{L} B_{x} B_{z} \sqrt{1-\beta_{L}^{2}}\right]$, 


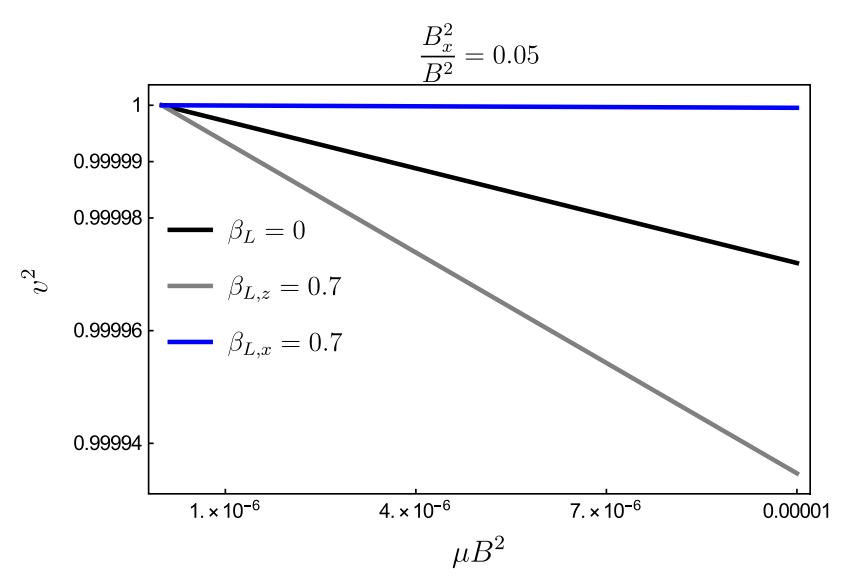

Fig. 5 The phase velocities for metric 1 are shown in three cases: $v_{1}$ for the metric Eq. (10) (black) and then the corresponding to the Lorentz boost along the $z$ (grey) and $x$ (blue) directions. The slowing down is enhanced for the boost along the propagation direction

where $\beta_{L}$ is the velocity of the Lorentz transformation along $x$-direction and $\gamma_{L}=1 / \sqrt{1-\beta_{L}^{2}}$. The effect of the retarding term is enhanced and the relative magnitude between the parallel and perpendicular components of the magnetic field plays a rol as well. In Fig. 5 are illustrated the velocities for metric 1 in three cases, $v_{1}$ corresponding to the metric Eq. (10) and then the ones with the Lorentz boost along the $z$ and $x$ directions. The direction of the Lorentz velocity is important: when $\beta_{L}$ is in the same direction than the propagation, the braking of the wave is more effective than if $\beta_{L}$ is perpendicular to the propagation. In the former case the wave slows down with a magnetic background less intense than for a still medium.

It deserves a further study the modeling of a moving medium and specifically the precise relationship between the medium and the velocity of the Lorentz tranformation. For the case of the electric background we guess a similar behaviour than the magnetic one when Lorentz boosted.

\section{Conclusions}

We present the solutions to the truncated Euler-Heisenberg Lagrangian that represent an electromagnetic wave propagating through an intense uniform magnetic or electric background; due to the wave-background interaction one electric longitudinal component, $\gamma E_{w}$, arises affecting the polarization.. The constant $\gamma$ depends on three parameters: the velocity of the propagation, the magnetic background and the $\mathrm{EH}$ parameter $\gamma(\beta, B, \mu)$; if the magnetic field is such that there is no component perpendicular or parallel to the propagating direction, $B_{\perp}=0$ or $B_{\|}=0$ this effect does not occur and $\gamma=0$.
By means of the NLED effective metric approach $[26,27$, 31], we obtain there is birefringence and we determine the two possible phase velocities of the propagating wave. For intense magnetic background fields, but such that $B / B_{c r}<<$ 1 the velocities of the propagation slow down, diminishing to the order of hundred thousandths as $B$ grows, this is illustrated in Fig. 2. The birefringence in the case of an electric uniform background is calculated as well.

By performing a Lorentz boost on the effective metric we model the situation of a flowing medium. By rescaling the coordinates of the effective metric it acquires the form of a PLG metric, where clearly can be identified the velocity of the propagating medium and the velocity of the perturbation through such a medium. We present as example the case of Lorentz boosts parallel and perpendicular to the propagating direction. The effect of slowing down the wave is more efficient for a parallel to the propagation Lorentz boost.

In summary we have analyzed the slowing down of an electromagnetic propagating wave under the effect of a very intense electromagnetic field background, in the context of the truncated to one-loop Euler-Heisenberg theory, or the Euler-Kockel Lagrangian, that takes into account in an effective way the vacuum polarization phenomenon. We used the effective metric approach and showed it is equivalent to the soft photon approximation. We find there is birefringence in both cases the electric and the magnetic background, the phase velocities of the propagation depend on its polarization.

Acknowledgements We thank the anonymous referee whose criticism lead to improve our paper. The work of E G-H has been sponsored by CONACYT-Mexico through the M.Sc. scholarship no. 485042. N. B. acknowledges partial financial support from CONACYT-Mexico through the project no. 284489 .

Data Availability Statement This manuscript has no associated data or the data will not be deposited. [Authors' comment: It is a theoretical work and the figures were generated analytically.]

Open Access This article is licensed under a Creative Commons Attribution 4.0 International License, which permits use, sharing, adaptation, distribution and reproduction in any medium or format, as long as you give appropriate credit to the original author(s) and the source, provide a link to the Creative Commons licence, and indicate if changes were made. The images or other third party material in this article are included in the article's Creative Commons licence, unless indicated otherwise in a credit line to the material. If material is not included in the article's Creative Commons licence and your intended use is not permitted by statutory regulation or exceeds the permitted use, you will need to obtain permission directly from the copyright holder. To view a copy of this licence, visit http://creativecomm ons.org/licenses/by/4.0/.

Funded by SCOAP ${ }^{3}$. 


\section{References}

1. W. Heisenberg, H. Euler, Folgerungen aus der diracschen theorie des positrons. Zeitschrift Für Physik 98(11-12), 714-732 (1936). English translation: Consequences of Dirac's Theory of Positrons, arXiv:physics/0605038

2. G.V. Dunne, The Heisenberg-Euler effective action: 75 years on. Int. J. Mod. Phys. Conf. Ser. 14, 42-56 (2012). https://doi.org/10. 1142/S2010194512007222. arXiv: 1202.1557

3. G.V. Dunne, Heisenberg-Euler Effective Lagrangians: basics and extensions, Ian Kogan Memorial Collection, "From Fields to Strings: Circumnavigating Theoretical Physics", pp 445-522 (2005), https://doi.org/10.1142/9789812775344-001. arXiv:hep-th/0406216v1

4. H. Gies, F. Karbstein, An addendum to the Heisenberg-Euler effective action beyond one loop. J. High Energy Phys. 03, 018 (2017)

5. F. Karbstein, All-loop result for the strong magnetic field limit of the Heisenberg-Euler effective Lagrangian. Phys. Rev. Lett. 122, $211602(2019)$

6. S.L. Adler, J.N. Bahcall, C.G. Callan, M.N. Rosenbluth, Photon splitting in a strong magnetic field. Phys. Rev. Lett. 25, 1061 (1970)

7. V.I. Ritus, Radiative corrections in quantum electrodynamics with intense field and their analytical properties. Ann. Phys. 69(2), 555$582(1972)$

8. H. Euler, B. Kockel, The scattering of light by light in the Dirac theory. Naturwiss 23, 246 (1935)

9. Z. Bialynicka-Birula, I. Bialynicki-Birula, Nonlinear effects in quantum electrodynamics. Photon propagation and photon splitting in an external field. Phys. Rev. D 2, 2341-2345 (1970)

10. T. Gold, Rotating neutron stars as the origin of the pulsating radio sources. Nature 218, 731-732 (1968)

11. M.G. Baring, Photon splitting and pair conversion in strong magnetic fields. AIP Conf. Proc. 1051, 53-64 (2008). arXiv:0804.0832

12. M. Born, L. Infeld, Foundations of the new field theory. Proc. R. Soc. Lond. A 144, 425-451 (1934)

13. S.I. Kruglov, On generalized Born-Infeld electrodynamics. J. Phys. A Math. Theor. 43, 375402 (2010)

14. J. Schwinger, On gauge invariance and vacuum polarization. Phys. Rev. 82, 664-679 (1951)

15. W. Dittrich, H. Gies, Light propagation in nontrivial QED vacua. Phys. Rev. 58, 025004 (1998)

16. W. Dittrich, H. Gies, Probing the Quantum Vacuum, Springer Tracts Modern Physics, 166 (Springer, Berlin, 2000)

17. M. Aaboud et al., Evidence for light-by-light scattering in heavyion collisions with the ATLAS detector at the LHC. Nat. Phys. 13, $852-858$ (2017)
18. ShZh Akhmadaliev et al., Experimental investigation of highenergy photon splitting in atomic fields. Phys. Rev. Lett. 89(6), 061802 (2002). arXiv:hep-ex/0111084

19. A.N. Luiten, J.C. Petersen, Detection of vacuum birefringence with intense laser pulses. Phys. Lett. A 330(6), 429-434 (2004)

20. G. Brodin, M. Marklund, L. Stenflo, Detection of QED vacuum nonlinearities in Maxwell's equations by the use of waveguides. Phys. Rev. Lett. 87, 171801 (2001)

21. F. Gelis, N. Tanji, Schwinger mechanism revisited. Prog. Part. Nucl. Phys. 87, 1 (2016)

22. I.A. Batalin, A.E. Shabad, Green's function of a photon in a constant homogeneous electromagnetic field of general form. Sov. Phys. JETP 33, 483-486 (1971)

23. E. Brezin, C. Itzykson, Polarization phenomena in vacuum nonlinear electrodynamics. Phys. Rev. D 3, 618-621 (1971)

24. F. Karbstein, Photon polarization tensor in a homogeneous magnetic or electric field. Phys. Rev. D 88, 085033 (2013)

25. M. Aiello, R.G. Bengochea, R. Ferraro, Anisotropic effects of background fields on Born-Infeld electromagnetic waves. Phys. Lett. A 361, 9-12 (2007)

26. S. Alarcon Gutierrez, A.L. Dudley, J.F. Plebański, Signals and dicontinuities in general relativistic non-linear electrodynamics. J. Math. Phys. 22, 2835-2848 (1981)

27. M. Novello, V.A. De Lorenci, J.M. Salim, R. Klippert, Geometrical aspects of light propagation in nonlinear electrodynamics. Phys. Rev. D 61, 045001 (2000)

28. V.A. De Lorenci, R. Klippert, M. Novello, J.M. Salim, Light propagation in nonlinear electrodynamics. Phys. Lett. B 482, 134-140 (2000)

29. Y.N. Obukhov, G.F. Rubilar, Fresnel analysis of wave propagation in nonlinear electrodynamics. Phys. Rev. D 66, 024042 (2002)

30. E. Goulart, S.E. Perez Bergliaffa, A classification of the effective metric in nonlinear electrodynamics. Class. Quantum Gravity 26, 13, 135015 (2009)

31. S. Liberati, S. Sonego, M. Visser, Scharnhorst effect at oblique incidence. Phys. Rev. D 63, 085003 (2001)

32. S. Hu, B. Liu, Birefringence and non-transversality of light propagation in an ultra-strongly magnetized vacuum. J. Phys. A Math. Theor. 40, 13859-13867 (2007)

33. H. Gies, Strong laser fields as a probe for fundamental physics. Eur. Phys. J. D 55, 311-317 (2009). arXiv:0812.0668

34. F. Karbstein, Probing vacuum polarization effects with highintensity lasers. Particles 3(1), 39-61 (2020)

35. R. Aguero-Santacruz, D. Bermudez, Hawking radiation in optics and beyond. Phil. Trans. R. Soc. A378, 20190223 (2020). https:// doi.org/10.1098/rsta.2019.0223. arXiv:2002.07907 [gr-qc] 